\title{
Modeling of High Speed Free Space Optics System to Maintain Signal Integrity in Different Weather Conditions; System Level
}

\author{
Rao Kashif \\ Department of Electronic Science and Technology \\ University of Science and Technology of China \\ Hefei, China \\ Oluwole John \\ Department of Electronic Science and Technology \\ University of Science and Technology of China \\ Hefei, China
}

\author{
Fujiang Lin \\ Department of Electronic Science and Technology \\ University of Science and Technology of China \\ Anhui, China \\ Abdul Rehman Buzdar \\ Department of Electronic Science and Technology \\ University of Science and Technology of China \\ Anhui, China
}

\begin{abstract}
Free space optical (FSO) also known as free space photonics (FSS) is a technology widely deployed in Local Area Network (LAN), Metro Area Network (MAN), and in Inter \& Intra chip communications. However satellite to satellite and other space use of FSO requires further consideration. Although FSO is highly beneficial due to its easy deployment and high security in narrow beam as well as market demand for $10 \mathrm{~GB}+$, some factors especially rain, snow and fog attenuation causes signal integrity problem in FSO. To get better Signal Integrity in FSO we need to consider all components while designing the system. In this paper a comparative analysis has been performed on $10 \mathrm{~GB}$ and 40 GB FSO system over $1 \mathrm{Km}$. Firstly for selecting suitable modulation technique we compared NRZ and RZ modulation and get spectrum analysis. NRZ modulation was found more data efficient. Signal Integrity in FSO system with 10 GB/s was analyzed by eye diagram and Q-Factor of both APD and PIN Photo detector was presented in graph. Same experiment was repeated with $40 \mathrm{~GB} / \mathrm{s}$ and Bit error rate of both photo detectors were presented.
\end{abstract}

Keywords-Free Space Optical; NRZ; RZ; PIN; APD; Photo Detector; BER; Q-factor

\section{INTRODUCTION}

Free space optical (FSO) is a transmission system which provide point to point, mesh and point to multi point communication by using laser and photodiodes. It can be a good candidate for high bandwidth future broadband and communication systems. Due to low BER, high bandwidth and easy installation FSO is popular in optical and wireless research community One more advantage of FSO is its Unlicensed Frequency Spectrum (800-1700nm) [1]. FSO is also a smart selection for intra satellite communication and due to small terminal and low power it has advantage over microwave links [2]. The first laser link to handle commercial traffic was built in Japan by Nippon Electric Company (NEC) around 1970. FSO is also efficient and being used for underwater communication, indoor wireless optical network, Intra chip to chip and board to board communication [3] and intra satellite communication [2].

Beside this, FSO also have number of challenges. One of those challenges is weather attenuation to optical signal. Rain, Snowfall and FOG are big challenges and they need researcher's consideration to maintain signal integrity in FSO system as the data-rate and coverage distance increases. To keep signal integrity throughout the system we need to consider few factors by selecting right blocks and components for modulation, receiver photodiodes, and Noise mechanism. In this paper we design a simple point to point FSO system in OpticSystem 14.0 and check signal integrity by PIN \& APD photodiode in different weather conditions with $10 \mathrm{~GB} / \mathrm{s}$ and $40 \mathrm{~GB} / \mathrm{s}$ data rate over $1 \mathrm{Km}$.

\section{NRZ AND RZ MODULATION TECHNIQUES}

Selecting right modulation technique which will convert electrical signal into bit stream is the first step for optical system designing. Using INTERCONNECT (Lumerical) with $25 \mathrm{~GB} / \mathrm{s}$ PRBS bit rate generator we compared NRZ (NonReturn to Zero) and RZ (Return to Zero) modulation as shown in Fig.1. NRZ modulation does not have rest state Fig.2a while signal drops to zero between each pulse in RZ Fig.2b. NRZ is more data efficient as it requires only half the bandwidth as compared to RZ Fig.3(a) \& (b). 


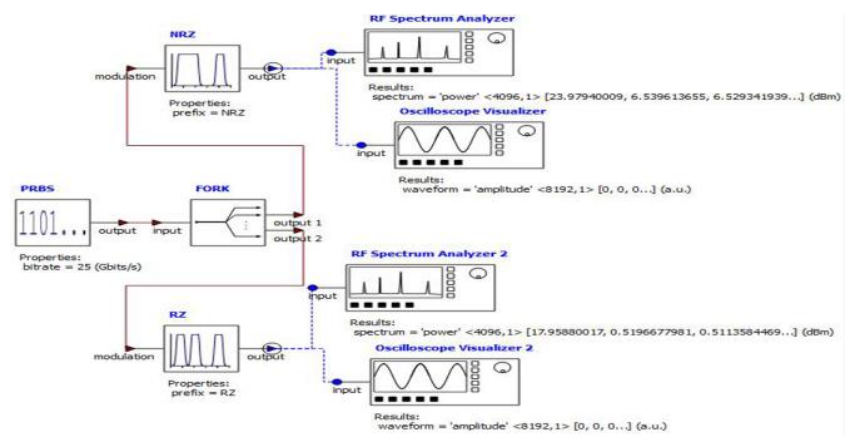

Fig. 1. NRZ \& RZ Modulation Technique

(a) NRZ

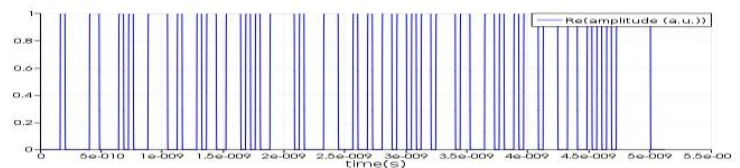

(b) $R Z$

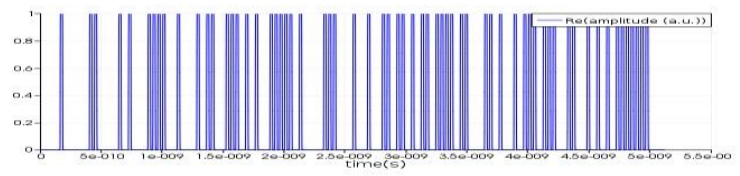

Fig. 2. NRZ \& RZ Oscilloscope analysis

(a) NRZ

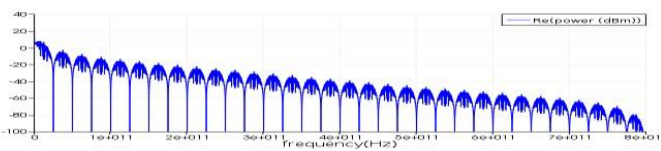

(b) RZ

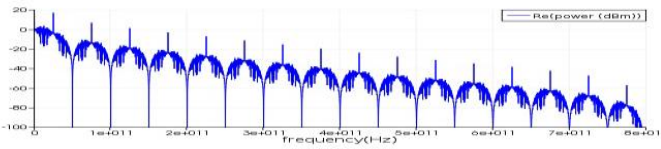

Fig. 3. NRZ \& RZ bandwidth spectrum analysis

\section{Attenuation in Free Space Optics System}

As FSO system uses free space for transmission medium so weather condition is one of the challenges which require consideration [4]. Even the clean state of atmosphere can't be refers as free space because Nitrogen and Oxygen are there. Attenuation cause to signal in atmosphere is called atmospheric attenuation [5]. Beer's Law [5] (1) is used unremarkably to relate atmospheric attenuation. PR is received optical power and $\operatorname{Pr}$ is optical power at source. $\gamma(\mathrm{L})$ is total attenuation [5].

$$
\tau(\lambda, L)=\frac{P_{R}}{P_{r}}=\exp (-\gamma(\lambda)) \cdot L
$$

Rain attenuation is one of the causes for atmospheric attenuation in tropical regions. Rayleigh, Mie and Non selective are there different types of atmospheric scattering. Redirection of light which leads to reduction of received light intensity is called scattering [6]. Non-selective scattering happens when rain drop size is larger than wavelength [7]. Absorption occurs by interaction between molecules and propagation photons in atmosphere [8]. The visibility range is the distance travel by beam till its intensity drop to $5 \%$ of its real [9].

\section{SimUlation SETUP WITH PIN AND APD PHOTO DETECTOR}

Photo detector is a device which converts optical signal to electrical signal. Photo Intrinsic Negative (PIN) and Avalanche Photo Diode (APD) are two photo detectors use in Free space Optic. The overview diagram of system which we design and use for comparison of APD and PIN photodiode is shown in Fig. 4. Simulation was done with $10 \mathrm{~GB}$ and $40 \mathrm{~GB}$ bit/s on distance of $1 \mathrm{~km}$ (1000 meter). NRZ modulation technique was used because its data efficiency is higher than RZ modulation as we compared it in Fig. 2 and Fig. 3. Shot noise and Thermal noise are two noise mechanisms in Photo detector. In Fig.5a we enable Shot noise in Photo detector and in Fig. 5b thermal noise were enabled. Due to high Q-factor we enabled thermal noise in our system. Laser frequency in system was $1550 \mathrm{~nm}$. As Power of system vary according to different weather conditions $(1-21 \mathrm{db})$. For tropical areas attenuation for haze and rain can be calculates by considering International visibility code refer from [10].Table 1 shows attenuation giving to the system.

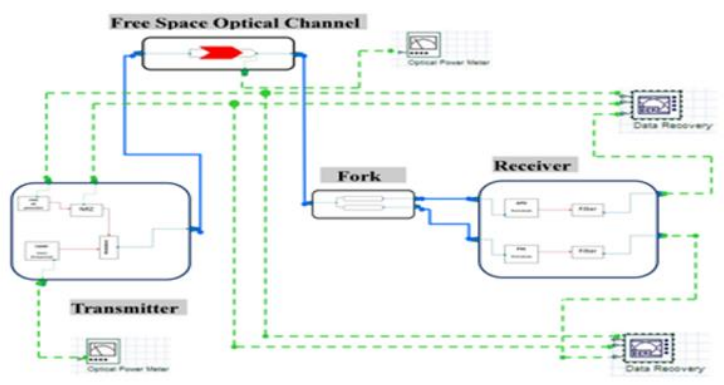

Fig. 4. System Overview Design

(a) Shot noise

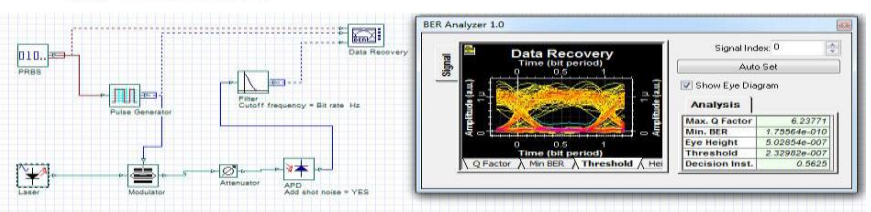

(b) Thermal noise

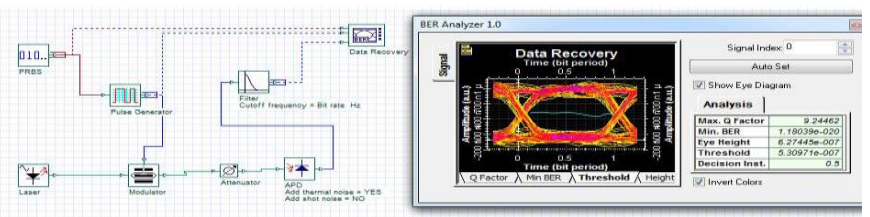

Fig. 5. Shot and Thermal noise Analysis 
TABLE. I. WEATHER ATTENUATION

\begin{tabular}{|l|l|l|}
\hline \multirow{2}{*}{ No. } & Weather conditions \\
\cline { 2 - 3 } & Weather Type & $\begin{array}{l}\text { Attenuation } \\
\text { db/Km }\end{array}$ \\
\hline 1 & Clear & 2.5 \\
\hline 2 & Haze & 4.35 \\
\hline 3 & Light rain & 12 \\
\hline 4 & High rain & 20 \\
\hline 5 & Snow & 25 \\
\hline
\end{tabular}

\section{PIN \& APD PHOTO DETECTOR Q-FACTOR ANALYSIS WITH $10 \mathrm{~GB}$}

Figure 6 shows system diagram for $10 \mathrm{~GB}$ data over $1 \mathrm{Km}$ Free Space Optical channel in OpticSystem 14.0. Different weather condition attenuation show in Table.1 was given to channel and EYE diagrams to get Q-factor analysis. Fig.7 shows result from APD photo detector and Fig.8 PIN photo detector. Q-Factor of both Photo detectors are analyzed and plotted in graph Fig.9. APD photo detector showed high QFactor as compared to PIN photo detector. As the attenuation increases the input power of system was also increased to maintain signal Integrity. Overall power for all weather conditions are plotted in Fig.10.

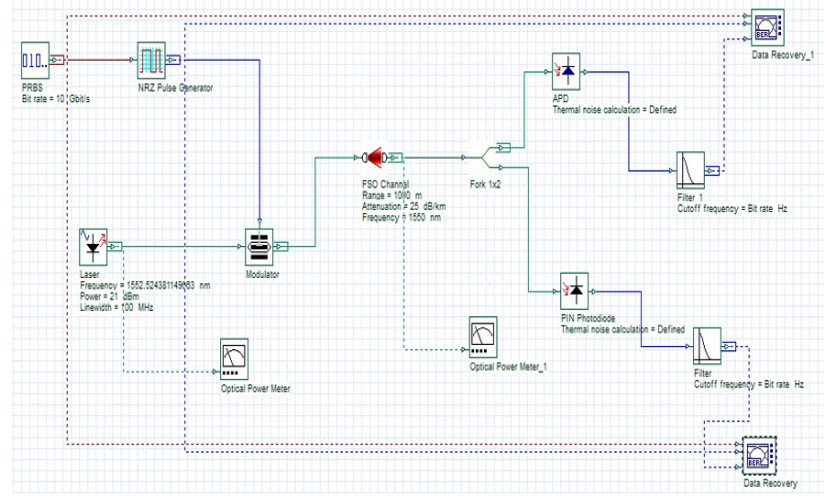

Fig. 6. System Diagram with $10 \mathrm{~Gb} / \mathrm{s}$

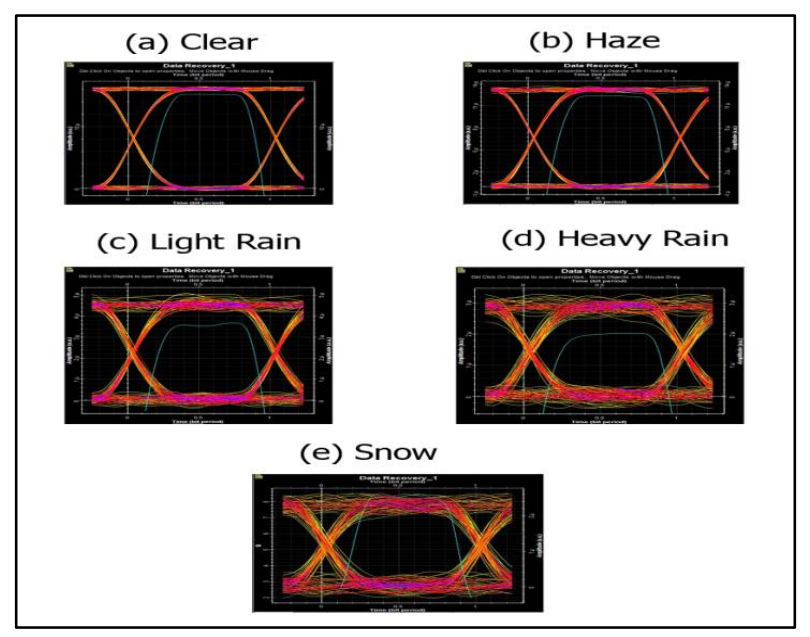

Fig. 7. APD Photo Detector EYE Analysis

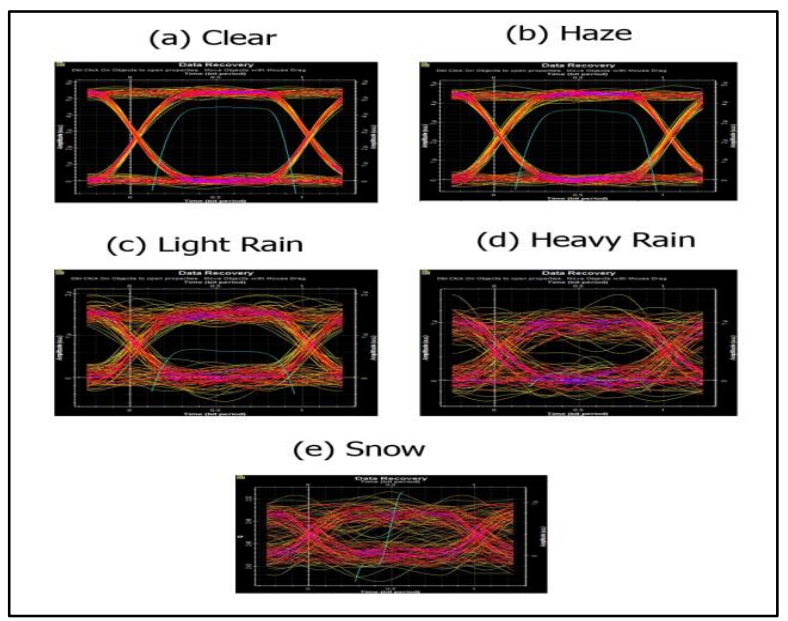

Fig. 8. PIN Photo Detector EYE Analysis

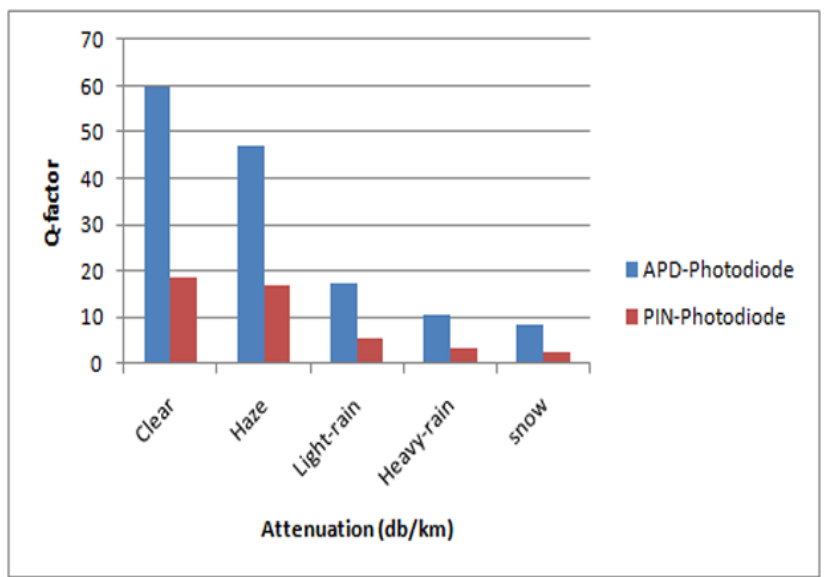

Fig. 9. PIN and APD photo detector Q-factor analysis

\section{PIN \& APD PHOTO DETECTOR BIT ERROR RATE (BER) ANALYSIS WITH $40 \mathrm{~GB}$}

Same system in Fig.6 was used for 40 GB data transfer over $1 \mathrm{Km}$ and signal Integrity was analyzed by analyzing Bit Error Rate (BER). Fig.11shows plotted graph of BER of APD and PIN photo detector. The whole system power increases with attenuation and increase in the data rate. Fig.12 graph shows over all power analysis for 10 and 40 GB in different weather conditions.

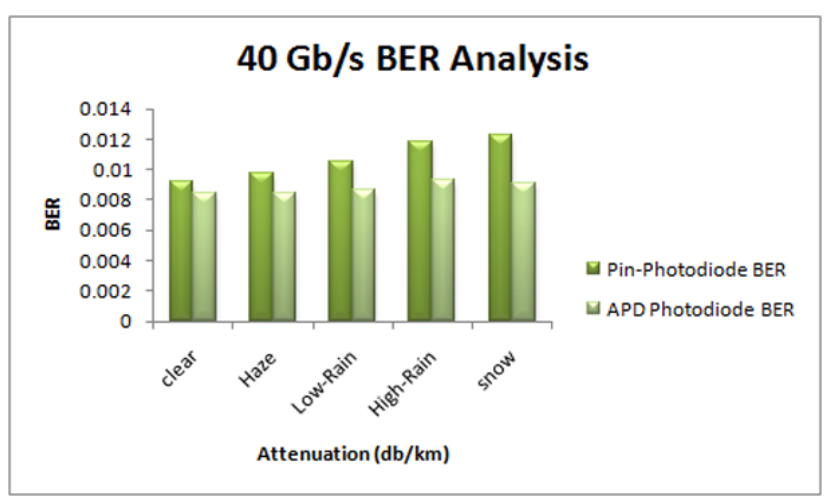

Fig. 10. APD and PIN photo detector BER Analysis 


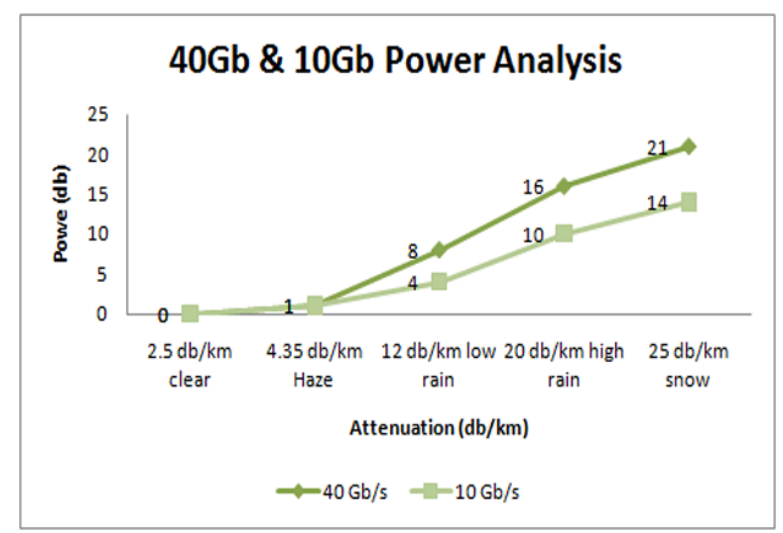

Fig. 11. Power analysis for 40 and $10 \mathrm{~Gb}$ system

\section{CONCLUSION}

Signal Integrity and performance comparison of APD and PIN photo detector were evaluated in FSO system. We concluded that APD have better performance than PIN photo detector, so optical receiver with APD photo detector provide better signal Integrity as compared to PIN. As BER can be decrease by increasing optical power so future experiments can be on different NRZ modulation techniques to get better power Integrity in FSO communication.

\section{ACKNOWLEDGMENT}

This research is supported by CAS-TWAS and Micro/Nano Electronic System Integration R\&D Center China. We thank our colleagues who provided insight and expertise that greatly assisted the research.

\section{REFERENCES}

[1] Arun.K, Advance Free Space Optics (FSO), Volume 186, Springer, CA 2014, pp.09.

[2] Amon.S, and Kopeika.N, The performance limitations of free space optical communication satellite networks due to vibrations- Analog case, Proceedings of the Electrical and Electronic Engineers in Israel, 1996, Nineteenth convention of, November 5-6, 1996, Jerusalem,Isreal,pp.287-290.

[3] Tsang.D, Free Space board to board optical Interconnection, Proceedings of SPIE 1563, Optical Enhancements to Computing Technology, December 1, 1991, University of Lowa, USA,pp.66-71.

[4] Zabidi.S, Islam.M, Khateeb.W, and Naji.A, Investigating of rain attenuation impact on Free Space Optics propagation in tropical region, Proceedings of Mechatronics (ICOM), 2011 4th International Conference On, May 17-19,2011, Kuala Lumpur, Malaysia,pp.1-6.

[5] Robert.K, Propagation handbook for wireless communication system design.Volume 1, CRC Press, Florida July 2003.

[6] Zhuanhong.J, Qingling.Z, Atmospheric Attenuation Analysis in the FSO link, Proceedings of International conference of Communication technology (ICCT’06), Nov 27-30 2006, Guilin, China.pp.1-4.

[7] Maha.A, Simulating atmospheric free space optical propagation, Proceedings of SPIE 4635, Free-Space Laser communication Technologies, April 2002, San Jose, California,pp.192-201.

[8] Popoola.W, Ghassemlooy.Z, Awan.M, and Leitgeb.E, Atmospheric Channel effects on terrestrial free space optical communication links, Proceedings of ECAI 2009 International Conference Electronics, Computers and Artificial Intelligence, July 3-5, Pitesti, Romania.pp.1723.

[9] Willebrand.H, Ghuman.H, Free space optics: enabling optical connectivity in today's network, edition 1, Sama Publications, Indiana 2001.

[10] ITU-R P.1814, R. (2007-08). "Prediction methods required for the design 